\title{
Retroperitoneoscopic distal pancreatectomy: a new surgical approach
}

\author{
Long-Zhi Zheng ${ }^{1}$, Guo-Wei Lin², Chang-Yue Zheng ${ }^{1}$, Jian Guo ${ }^{1}$, Bin Zu ${ }^{1}$, Jian-Xin Huang ${ }^{3}$, Wei Lin ${ }^{1}$ \\ ${ }^{1}$ Department of Gastrointestinal Surgery and Gastrointestinal Surgery Research Institute, the Affiliated Hospital of Putian University, \\ Putian, China \\ 2Department of Urinary Surgery, the Affiliated Hospital of Putian University, Putian, China \\ ${ }^{3}$ Department of Ultrasonography, the Affiliated Hospital of Putian University, Putian, China
}

Videosurgery Miniinv 2019; 14 (3): 374-380

DOI: https://doi.org/10.5114/wiitm.2019.81442

\begin{abstract}
Introduction: The traditional laparoscopic surgery is difficult to deal with the deep lesions of the body and tail of the pancreas, which may damage the visceral organs of the abdominal cavity and cause abdominal adhesion and other related complications.

Aim: This paper introduces the operation procedure of retroperitoneoscopy in pancreatic surgery, and evaluates its feasibility in clinical application.

Material and methods: Retrospective analysis was performed on patients with retroperitoneal pancreatectomy in our hospital. The anatomical features of the fascia, surgical plane composition and surgical pathway of the fascia of the retroperitoneoscopic pancreatectomy were observed during the operation, and the surgical safety and feasibility were analyzed. The following parameters were evaluated: operation time, blood loss, pancreatic fistula, postoperative gastro-intestinal recovery, hospital stay.

Results: All 3 patients had a smooth operation and no serious complications occurred. During retroperitoneal laparoscopic pancreatectomy, there is a vascularized plane between the posterior fascia of the pancreas and the prerenal fascia, which can avoid injury of the visceral organs and retroperitoneal vessels. The anterior renal fascia should be used as the posterior boundary of the safe separation plane.

Conclusions: The surgical plane based on the anatomy of the fascia and interstitial dissection is the theoretical basis of modern surgery, which is safe, fast and effective. The inter-prerenal fascia plane is the correct and safe anatomical plane of posterior laparoscopic surgery.
\end{abstract}

Key words: pancreas, retroperitoneoscopy, inter-prerenal-fascia planes, Toldt's fascia.

\section{Introduction}

Distal pancreatectomy is the main surgical treatment for tumors in the body or tail of the pancreas, and in the early stage, it is mainly traditional open surgery. In recent years, with the rapid development of laparoscopic minimally invasive technique, there are currently some pancreatic procedures that can be performed under laparoscopic surgery, such as laparoscopic enucleation (LEn) of pancreatic tumors, laparoscopic distal pancreatectomy (LDP) (splenic preservation or splenectomy), robot-assisted distal pancreatectomy, and so on. Although pancreatic surgery is difficult and complex compared with urology and gynecology, the development of laparoscopic techniques is relatively slow. However, with the con-

\section{Address for correspondence}

Wei Lin PhD, Department of Gastrointestinal Surgery and Gastrointestinal Surgery Research Institute, the Affiliated Hospital of Putian University, 999 Dong Zhen Road, 351100 Putian, Fujian, China, phone: +86 05942730373 , e-mail: linwbj@outlook.com 
tinuous maturity and progress of surgical techniques and equipment in the past decade, laparoscopic pancreatic surgery, especially robot-assisted surgery, has been increasingly applied in pancreatic surgery with its unique advantages [1].

Schmidt et al. reported that the incidence of pancreatic tumors was increasing in recent years, and surgery is still the best treatment for pancreatic tumors [2]. The traditional transperitoneal laparoscopic method is difficult to expose the lesions deep in the body or tail of the pancreas. It is necessary not only to open the gastrocolonic ligament, but also to break the splenocolonic ligament, the gastrocolonic ligament and the lower margin of the pancreas and flip it up in order to reveal the lesions. The operation not only destroyed the original abdominal ligament and fascia, but also easily damaged the middle colic artery, colon and splenic vein. Especially in obese subjects or those with previous history of upper abdominal surgery, the difficulty of the laparoscopic operation increased significantly, and also the risk of the operation. For this purpose, the clinic is constantly trying to improve the operation and finding new surgical methods. It is expected to simplify the operation, reduce the difficulty and improve safety while preserving the minimally invasive characteristics, so as to further promote the development of minimally invasive surgery of the pancreas.

At present, the retroperitoneal laparoscopic adrenalectomy has become mature. Like the retroperitoneal organ, the pancreas is adjacent to the left adrenal gland, and the exposure of the posterior rear of the pancreas is basically consistent with adrenal surgery. The evolution of the adrenal surgical procedure (traditional laparotomy $\rightarrow$ transperitoneal laparoscopy $\rightarrow$ retroperitoneal laparoscopy) has some implications for pancreatic surgery. Here we introduce a new surgical approach, with reference to retroperitoneal laparoscopic adrenalectomy, followed by retroperitoneoscopic distal pancreatectomy. Our preliminary results show that retroperitoneoscopic distal pancreatectomy is a feasible surgical approach, which has the advantages of less trauma and rapid postoperative recovery. This suggests it may be a better surgical option for distal pancreatectomy.

\section{Aim}

The aim of the study was to introduce the operation procedure of retroperitoneoscopy in pancreatic surgery, and to evaluate its feasibility in clinical application.

\section{Material and methods}

This study was an observational study conducted between January 2016 and December 2017 in our hospital. The study included patients who underwent retroperitoneoscopic distal pancreatectomy in our hospital during this time period, with a total of 3 patients assessed during this period.

\section{Patients}

Conditions for patients to enter the group: Preoperative magnetic resonance imaging (MRI) showed that the lesions were located in the body or tail of the pancreas, benign or low-grade malignant tumor with a diameter of $\leq 5 \mathrm{~cm}$, no vascular invasion, a clear space with surrounding tissue, and no need to dissect lymph nodes (Photo 1). At the same time, patients had no history of retroperitoneal surgery.

Prior informed consent was signed by each patient and/or direct relative, and the hospital ethics committee approved the surgery.

\section{Surgical technique}

All patients underwent general anesthesia tracheal intubation, and the right lateral position was taken after anesthesia. The position was selected and the waist bridge was raised with the help of a urologist in order to maximize the space between the iliac crest and the costal margin (Photo 2). Three trocar holes were used to establish the space for the retroperitoneal operation. Port $A$ (under the costal margin of the posterior line axillary): a $2 \mathrm{~cm}$ skin

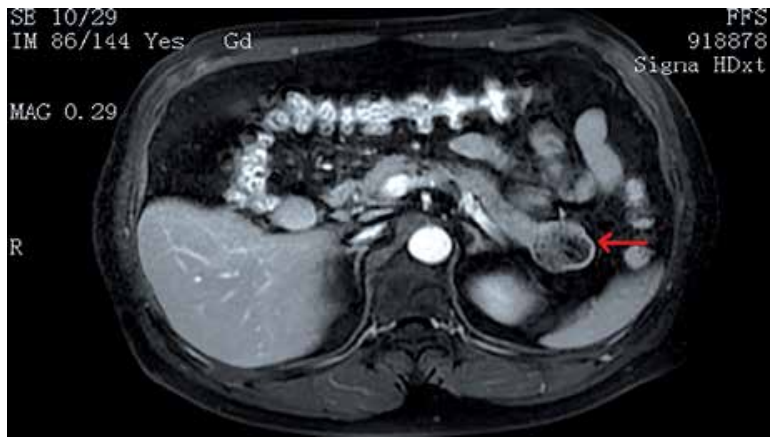

Photo 1. Magnetic resonance imaging scan showed that the tumor was located in the tail of the pancreas, about $2.5 \mathrm{~cm}$ in size, and the gap with the surrounding tissue was clear (arrow) 


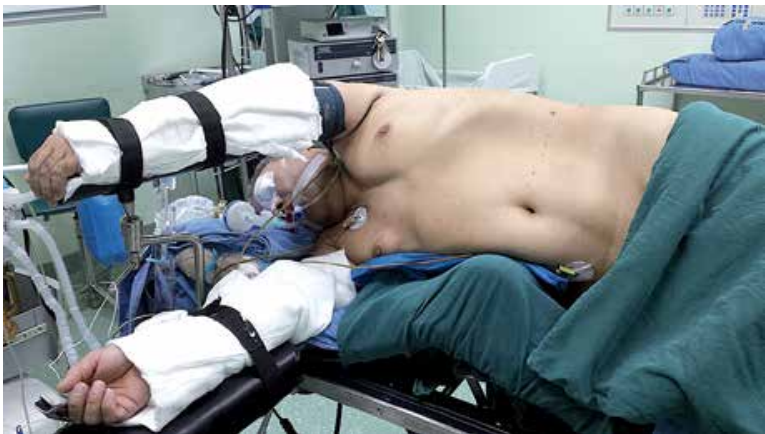

Photo 2. Surgical position: position on the right side, elevate the waist bridge. This position can maximize the space between the iliac crest and the costal margin

incision was made below the costal margin about $1 \mathrm{~cm}$ on the posterior axillary line. The muscles of the waist back and lumbodorsal fascia was bluntly separated by a vascular clamp, and the retroperitoneal space was penetrated and further separated by the forefinger. A self-made water sac was then placed through the incision. The water sac was filled with $300-500 \mathrm{ml}$ of water to expand the retroperitoneal space, which was maintained for 3-5 min to compress the capillary hemostasis and establish the retroperitoneal space. This port was placed with a $12 \mathrm{~mm}$ trocar and a harmonic scalpel as the main operating hole. Port B: at $2 \mathrm{~cm}$ above the junction of the midaxillary line and the iliac crest, a $10 \mathrm{~mm}$ trocar was used for the laparoscope as the observation hole. Port C: under the direct view of the laparoscope, a $5 \mathrm{~mm}$ trocar was inserted below the costal margin on anterior axillary line at about $2 \mathrm{~cm}$;

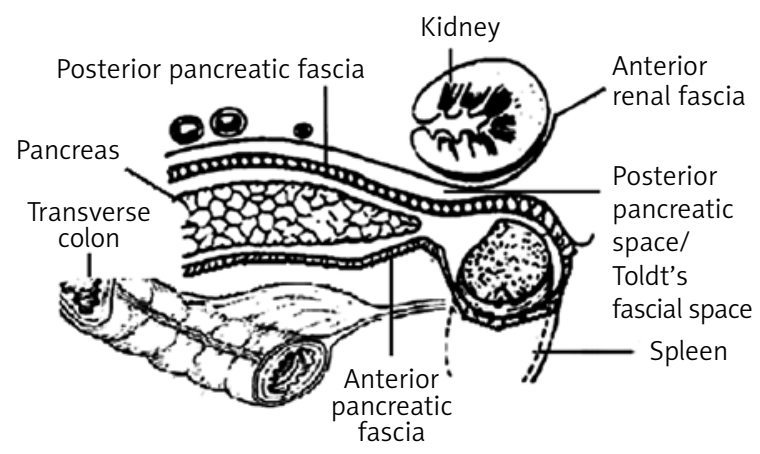

Figure 1. Anatomy of the pancreas around the pancreas. The interfascial plane of Toldt was the dissection plane for retroperitoneoscopic distal pancreatectomy, which was a security plane for pancreas surgery

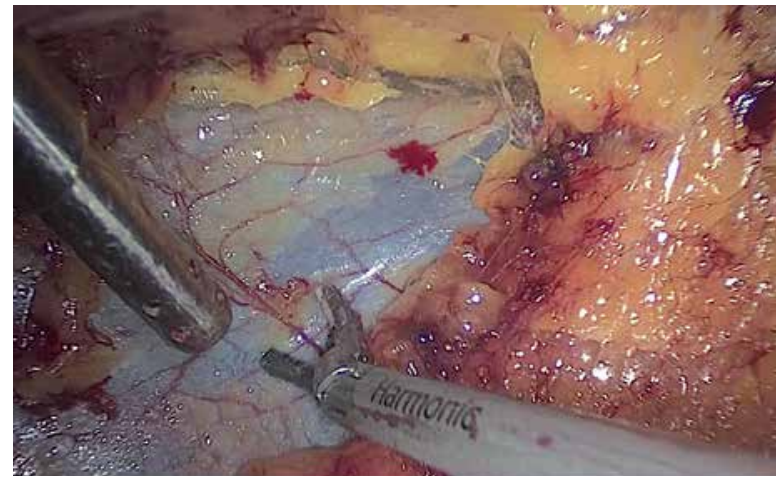

Photo 3. To clean up the extraperitoneal adipose tissue, expose the lateral conal fascial plane (LCP)

it was about the umbilical level. The corresponding devices were inserted into this port. The three points are roughly equal to an equilateral triangle, which is convenient for laparoscopic surgery. Carbon dioxide was then filled to maintain the surgical space, and the pressure was maintained at 12-14 $\mathrm{mm} \mathrm{Hg}$.

The extraperitoneal fat was freed from top to bottom with a harmonic scalpel and turned down to the iliac fossa in order to obtain more working space and achieve better anatomical guidance. After cleaning the extraperitoneal adipose tissue (Photo 3), the lateral conal fascial plane (LCP) can be clearly revealed. Then the lateral conal fascia (LCF) was identified and incised longitudinally near the retroperitoneal fold. Subsequently, the plane between the colonal fusion fascia and prerenal fusion fascia (PCPF) was reached and dissociated from the diaphragmatic peritoneum above the splenocolic ligament down to the iliac vessel level of the mesocolon sigmoideum. The ventral side was completely emptied by the gravity of the gut itself in the complete lateral position and the extrusion of $\mathrm{CO}_{2}$ during pneumoperitoneum. Then the prerenal fusion fascia plane (PRP) was dissociated in order to free the front of the prerenal fusion fascia (PRF). It is gently separated to enter into the gap of Toldt's fascia that was free of blood vessels and full of loose connective tissue, namely into the interfascial plane of Toldt (Figure 1), so that the integrity of the mesocolon and the anterior renal fascia can be retained, and a rarely bleeding operation can be achieved. Continue to expand into the posterior pancreatic space, and then the tumor located in the body or tail of the pancreas can be found (Photo 4). Subsequently, the pancreatic membrane was dissociat- 


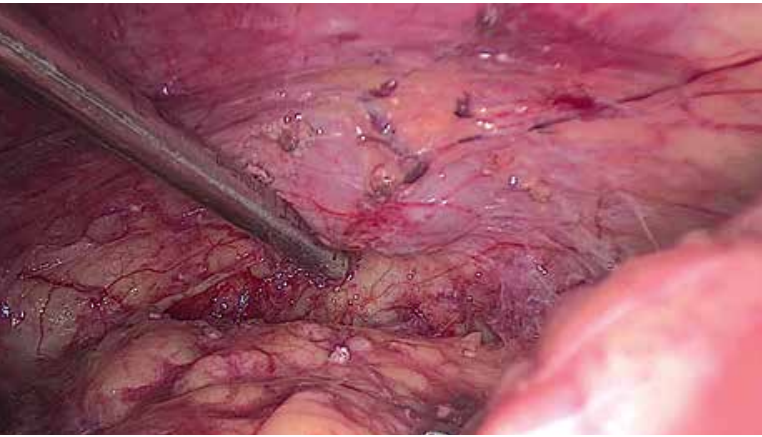

Photo 4. The tumor is located in the back of the pancreatic tail, which is more favorable for retroperitoneoscopic pancreatectomy

ed along the surface of the pancreas to expand the anterior pancreatic space (Photo 5). Then the splenic vessels were separated in detail, and the body/tail of the pancreas was fully exposed. Finally, the distal pancreas was cut off by an ENDOPATH Stapler Endoscopic Linear Cutter (Ethicon Endo-Surgery) (Photo 6). The specimen was removed from Port A using a selfmade specimen bag. Furthermore, double cannula drainage was placed in the wound, which was derived from Port $B$ and fixed.

\section{Results}

As shown in Table I, the operations of the three patients were successfully completed. The average operation time was about 140 min (range: 135-150 $\min$ ). Due to the direct exploration of the pancreas by retroperitoneal laparoscopic surgery, the abdominal viscera was interfered slightly and the patients could eat semi-liquid on the first day after the operation. The postoperative recovery was fast, and there were no obvious complications (pancreatic fistula, hemorrhage, etc.). The patients were discharged successfully and no recurrence was found after 3 months' follow-up.

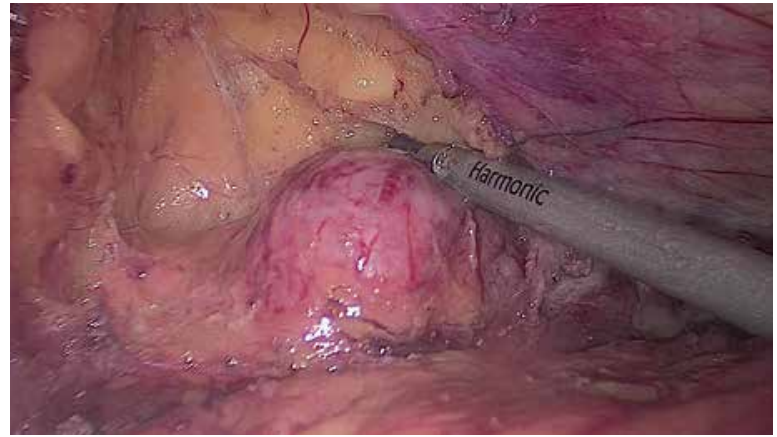

Photo 5. Fully dissociate the anterior pancreatic space and carefully protect the blood vessels of the spleen

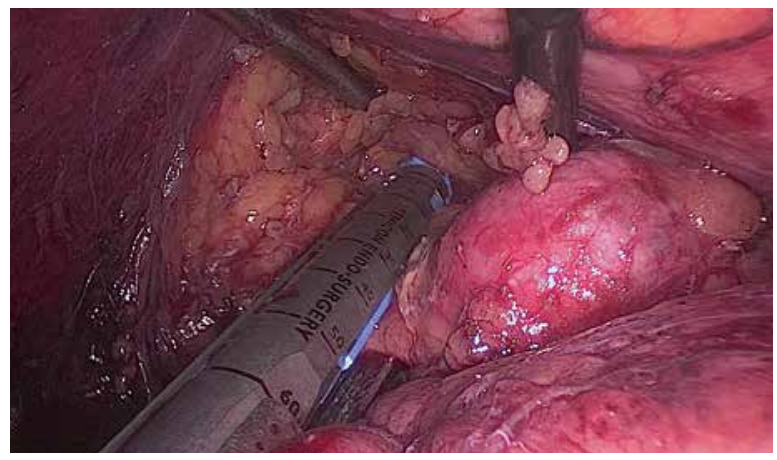

Photo 6. After fully dissociating the pancreatic tail, use a linear cutter stapler to cut off the distal pancreas

As shown in the photos, during the retroperitoneoscopic pancreatic surgery, we found that the inter-prerenal-fascia planes were anatomically communicated and embryologically homologous, forming a multiple-layered and avascular natural surgical plane in the anterior space of the kidney. The interfascial plane of Toldt was filled with spider silk lattice-like white loose fibrous tissue, which was a security plane for pancreas surgery. Through this plane, pancreas can be reached directly, and can achieve

Table I. Outcomes of patients undergoing retroperitoneoscopic distal pancreatectomy

\begin{tabular}{|cccccccc|}
\hline No. & Age/Sex & $\begin{array}{c}\text { Operation } \\
\text { time [min] }\end{array}$ & Blood loss [ml] & $\begin{array}{c}\text { Pancreatic } \\
\text { fistula }\end{array}$ & $\begin{array}{c}\text { Postoperative } \\
\text { gastro- } \\
\text { intestinal } \\
\text { recovery [h] }\end{array}$ & $\begin{array}{c}\text { Hospital stay } \\
\text { [day] }\end{array}$ & Pathology \\
\hline 1 & $58 / \mathrm{M}$ & 150 & 20 & No & 10 & 7 & SMA \\
\hline 2 & $61 / \mathrm{F}$ & 135 & 15 & No & 12 & 8 & Pancreatic cyst \\
\hline 3 & $65 / \mathrm{F}$ & 140 & 20 & No & 8 & 7 & $\begin{array}{c}\text { Pancreatic } \\
\text { pseudocyst }\end{array}$ \\
\hline
\end{tabular}

$M$ - male, F-female, SMA - serous microcystic adenoma of pancreas. 
a bloodless anatomy, which could significantly reduce erroneous dissection during laparoscopic surgery.

\section{Discussion}

In 1913, Mayo [3] first reported 3 cases of open distal pancreatectomy, in which the spleen was removed at the same time. In the following decades, distal pancreatectomy combined with splenectomy became the mainstream surgical approach for tumors in the body or tail of the pancreas. In 1982, Robey et al. analyzed the case data of distal pancreatectomy with splenic preservation [4]. Since then, more and more studies have shown that preservation of the spleen is very important to maintain the patient's immune function, and may even affect the patient's long-term prognosis [5, 6]. At this point, it has become routine for pancreatic surgery to preserve the spleen as much as possible. In 1994, Gagner and Pomp first reported the laparoscopic pancreatic surgery [7]. Because simple distal pancreatectomy does not require digestive tract reconstruction and there are fewer adjacent vessels, the application of laparoscopic techniques in the resection of the distal pancreas is becoming more and more extensive. A total of 806 cases of laparoscopic pancreatic surgery were reported in PubMed and Ovid databases up to 2009 [8]. Melvin et al. of Ohio State University reported the first case of robot-assisted distal pancreatectomy in 2002 [9]. At present, the robot-assisted surgical system is gradually becoming a new choice for pancreatic surgery, and it has been gradually carried out in multiple centers at home and abroad [10-12].

In 1969, Bartel [13] used an endoscope to detect retroperitoneal lesions from the retroperitoneum, which was later considered to be the originator of retroperitoneal laparoscopy. After that, Fantoni et al. [14] tried and evaluated the retroperitoneal laparoscopic nephrectomy, and made remarkable contributions to the development of retroperitoneal laparoscopic surgery, such as the establishment of the retroperitoneal space. In 1992, Gaur [15] first used the balloon dilation method and successfully established the retroperitoneal space, which made retroperitoneal laparoscopic surgery possible. In 2004, Molina et al. [16] reported a case of retroperitoneoscopic laparoscopic radical nephrectomy with concomitant distal pancreatectomy for a large renal mass with involvement of the left adrenal gland and the distal pancreas. This is also the earliest clinical application of retroperitoneal laparoscopy in pancreatic surgery.

In view of the difficulty of traditional transperitoneal laparoscopic surgery in revealing deep lesions of the body or tail of the pancreas, and the greater interference with the abdominal viscera, especially in patients with obesity or previous history of upper abdominal surgery, the difficulty of the transperitoneal approach was significantly increased. The retroperitoneal laparoscopy can avoid some of the risks of the above operation, and its biggest advantage lies in the direct exploration of the pancreas, with minimal interference to abdominal organs. In this study, our surgical approach was performed by retroperitoneal laparoscopy. It was found that there was a non-vascular plane between the posterior pancreatic fascia and the anterior renal fascia. The operation in this plane can prevent damage to the retroperitoneal vessels and intra-abdominal viscera, and the operation can be completed safely, quickly and effectively. This plane is the inter-prerenal-fascia plane. Therefore, the anterior renal fascia can be used as the posterior boundary of the surgical separation plane, and the inter-prerenal-fascia plane is the correct and safe anatomical plane for the retroperitoneoscopic distal pancreatectomy.

The key to the success of this operation is how to find the anatomical plane correctly. According to the developmental characteristics of embryology, the pancreas occurs at the $5^{\text {th }}$ week of the embryo and originates from the foregut endoderm and is divided into the ventral and dorsal pancreas. Thereafter, the dorsal peritoneum of the pancreas gradually merges with the posterior peritoneum, making it an extraperitoneal organ. Therefore, there is an avascular loose space between the dorsal pancreas and the posterior peritoneum, namely the posterior pancreatic space, which extends downward and communicates with the interfascial plane of Toldt. We define this plane as the surgical plane. It is a natural avascular zone filled with loose connective tissue, which facilitates tissue separation and keeps the surgical field clear. In the course of this surgery, we mainly divide it into two stages. First, after we have established the retroperitoneal space and cleaned up the extraperitoneal adipose tissue, the plane of the lateral conal fascia was clearly exposed. Then we could enter the inter-prerenal fascia plane after the incision of the lateral conal fascia. The completion 
of the entry from the lateral conal fascial plane to the correct inter-prerenal fascia plane was the key first step in the retroperitoneoscopic distal pancreatectomy, which we called the first dissection plane. Second, after correctly entering the inter-prerenal fascia plane, we could enter the interfascial plane of Toldt, which we called the second dissection plane, as we continued to dissociate the anterior surface of the prerenal fusion fascia. Behind the anterior renal fascia, the left ureter can be clearly seen and extended upward along this plane to enter the posterior pancreatic space and further expand the anterior pancreatic space; thus the pancreatic body and tail can be clearly displayed in the surgical field.

In terms of the surgical detail, because the interprerenal-fascia is a multiple-layered fascia, it is a thin membrane structure, and how to maintain its integrity is the tricky part of the surgery. As we know, the anterior renal fascia and the posterior renal fascia fuse at the lateral side of the kidney to form the lateral conic fascia, which is then attached to the peritoneum of the paracolonic sulci (that is, the peritoneal reflex) at the rear of the ascending or descending colon, so the lateral conus fascia should be cut longitudinally along the peritoneal reflex line, so that we can enter the first plane smoothly. In the process of the operation, in addition to being fully familiar with retroperitoneal anatomic landmarks, the surgical team must cooperate with each other. The mirror holder and the first assistant should assist the principal surgeon to maintain the proper tension in the surgical plane, because usually there is a lot of loose connective tissue between different planes, and maintaining the proper tension is conducive to the exposure and correct anatomy of the interplanar space. In addition, blunt dissection and sharp dissection should be combined in the separation of the gaps in each plane, so as to make the surgical field bloodless and clean. And one should avoid damaging the peritoneum during the operation, because peritoneal damage can lead to gas entering the abdominal cavity, which will make the space of the retroperitoneal cavity smaller and the operation more difficult.

In the clinical practice of this operation, especially for beginners, it is easy to deviate from the anatomic plane, causing peritoneal rupture into the abdominal cavity, or damage the blood vessels and tissues behind the retroperitoneum, resulting in accidental hemorrhage or surgical failure. Therefore, the selection of appropriate cases, accurate preoperative positioning of the lesions, and mastering the retroperitoneal anatomical landmarks is the key to the success of this procedure. Of course, in the initial development one needs to learn more from urologists who have the experience of retroperitoneal laparoscopy and complete it together, which can shorten the learning curve and allow one to grasp the skills of retroperitoneal laparoscopic surgery more quickly.

The main postoperative complication of pancreatic surgery is pancreatic fistula. According to literature reports, the incidence of postoperative pancreatic fistula is about $4-45 \%$ in patients undergoing laparoscopic distal pancreatectomy [17-19], which is equivalent to open surgery. The 3 patients who underwent this operation recovered well after surgery, and no complications of pancreatic leakage or death occurred. Of course, this is related to the small number of patients who choose to undergo retroperitoneoscopic distal pancreatectomy at present, and the incidence of postoperative pancreatic leakage needs more clinical studies to demonstrate. The procedure of retroperitoneoscopic distal pancreatectomy also needs to be improved by surgeons in clinical practice.

\section{Conclusions}

Our preliminary results show that the retroperitoneoscopic distal pancreatectomy is a feasible surgical approach, which has the advantages of a direct approach, less interference of the abdominal organs, fewer surgical complications and rapid postoperative recovery. In particular, for obese patients or those with a previous history of upper abdominal surgery, the retroperitoneoscopic surgical approach may be a better surgical option. Also, this kind of operation is more in line with the modern concept of ERAS [20], which can reduce surgical stress, maintain a stable internal environment, speed up postoperative rehabilitation, shorten hospital stay and reduce hospitalization expenses. In addition, with the continuous improvement of surgical medical technique and medical equipment, as well as the development of the multidisciplinary team (MDT), the application of retroperitoneal laparoscopy in pancreatic surgery will gradually become mature.

\section{Acknowledgments}

This study was sponsored by the Natural Science Foundation of Fujian Province, Fund Project is "SPERT regulates cell proliferation and apoptosis 
of colorectal cancer through the MAPK signaling pathway", the Campus Scientific Research Projects of Putian University (No. 2017078) and the Science and Technology Foundation of the Education Department of Fujian Province (No. JAT170520).

\section{Conflict of interest}

The authors declare no conflict of interest.

\section{References}

1. Alsfasser G, Hermeneit S, Rau BM, et al. Minimally invasive surgery for pancreatic disease - current status. Dig Surg 2016; 33: 276-83.

2. Schmidt CM, Powell ES, Yiannoutsos CT, et al. Pancreaticoduodenectomy: a 20-year experience in 516 patients. Arch Surg 2004; 139: 718-27.

3. Mayo WJ. I. The Surgery of the pancreas: I. Injuries to the pancreas in the course of operations on the stomach. II. Injuries to the pancreas in the course of operations on the spleen. III. Resection of half the pancreas for tumor. Ann Surg 1913; 58: 145-50.

4. Robey E, Mullen JT, Schwab CW. Blunt transection of the pancrease treated by distalpancreatectomy, splenic salvage and hyperalimentation. Four cases and review of the literature. Ann Surg 1982; 196: 695-9.

5. Shoup M, Brennan MF, McWhite K, et al. The value of splenic pres ervation with distal pancreatectomy. Arch Surg 2002; 137: 164-8.

6. Linet MS, Nyrén O, Gridley G, et al. Causes of death among patients surviving at least one year following splenectomy. Am J Surg 1996; 172: 320-3.

7. Gagner M, Pomp A. Laparoscopic pylorus-preserving pancreatoduodenectomy. Surg Endosc 1994; 8: 408-10.

8. Borja-Cacho D, Al-Refaie WB, Vickers SM, et al. Laparoscopic distal pancreatectomy. J Am Coll Surg 2009; 209: 758-65.

9. Melvin WS, Needleman BJ, Krause KR, et al. Computer-enhanced robotic telesurgery. Initial experience in foregut surgery. Surg Endosc 2002; 16: 1790-2.

10. Waters JA, Canal DF, Wiebke EA, et al. Robotic distal pancreatec tomy: cost effective? Surgery 2010; 148: 814-23.

11. Ntourakis D, Marzano E, De Blasi V, et al. Robotic left pancreatectomy for pancreatic solid pseudopapillary tumor. Ann Surg Oncol 2011; 18: 642-3.

12. Pericleous S, Middleton N, McKay SC, et al. Systematic review and meta-analysis of case-matched studies comparing open and laparoscopic distal pancreatectomy: is it a safe procedure? Pancreas 2012; 41: 993-1000.

13. Bartel M. Retroperitoneoscopy. An endoscopic method for inspection and bioptic examination of the retroperitoneal space. Zentralbl Chir 1969; 94: 377-83.

14. Fantoni PA, Tognoli S, Astuni M, et al. A technique of low retroperitoneoscopy. Endoscopy 1982; 14: 102-4.

15. Gaur DD. Laparoscopic operative retroperitoneoscoppy: use of a device. J Urol 1992; 148: 1137-9.

16. Molina WR, Desai MM, Ng CS, et al. Retroperitoneoscopic radical nephrectomy with concomitant distal pancreatectomy: case report. J Endourol 2004; 18: 665-7.
17. Fernández-Cruz L, Cosa R, Blanco L, et al. Curative laparoscopic resection for pancreatic neoplasms: a critical analysis from a single institution. J Gastrointest Surg 2007; 11: 1607-21.

18. Taylor C, O'Rourke N, Nathanson L, et al. Laparoscopic distal pancreatectomy: the Brisbane experience of forty-six cases. HPB (Oxford) 2008; 10: 38-42.

19. Sa Cunha A, Rault A, Beau C, et al. A single-institution prospective study of laparoscopic pancreatic resection. Arch Surg 2008; 143: 289-95.

20. Kehlet H. Multimodal approach to control postoperative pathophysiology and rehabilitation. Br J Anaesth 1997; 78: 606-17.

Received: 1.10.2018, accepted: 5.12.2018. 